\title{
CORRESPONDENCES OF THE GELFAND INVARIANTS IN REDUCTIVE DUAL PAIRS
}

\author{
MINORU ITOH \\ (Received 15 May 2001; revised 27 November 2002)
}

Communicated by R. B. Howlett

\begin{abstract}
For each complex reductive dual pair introduced by $R$. Howe, this paper presents a formula for the central elements of the universal enveloping algebras given by I. M. Gelfand. This formula provides an explicit description of the correspondence between the 'centers' of the two universal enveloping algebras.
\end{abstract}

2000 Mathematics subject classification: primary 17B35; secondary 15A33.

Keywords and phrases: center of universal enveloping algebra, Gelfand invariants, dual pair.

\section{Introduction}

For each complex reductive dual pair introduced in [5], the images of the 'centers' of the two universal enveloping algebras coincide. In this paper we explicitly describe this correspondence between the universal enveloping algebras in terms of the central elements due to I. M. Gelfand.

Throughout this paper we will work over the complex number field $\mathbb{C}$. Let $\left(G, G^{\prime}\right)$ be a reductive dual pair in the complex symplectic group $S p_{N}=S p_{N}(\mathbb{C})$. Namely $G$ and $G^{\prime}$ are reductive subgroups of $S p_{N}$ such that each is the centralizer of the other in $S p_{N}$. Then, under the oscillator representation $\omega$ of $\mathfrak{s p}_{N}$, the images of the invariants with respect to the adjoint actions of $G$ and $G^{\prime}$ on the universal enveloping algebra $U\left(\mathfrak{s p}_{N}\right)$ are generated by the images of the Lie algebras $\mathfrak{g}^{\prime}=\operatorname{Lie}\left(G^{\prime}\right)$ and $\mathfrak{g}=\operatorname{Lie}(G)$, respectively ([5]). In particular, we have the following equality between the two universal enveloping algebras:

$$
\omega\left(U(\mathfrak{g})^{G}\right)=\omega\left(U\left(\mathfrak{g}^{\prime}\right)^{G^{\prime}}\right) .
$$

The author is partially supported by JSPS Research Fellowships for Young Scientists.

(C) 2003 Australian Mathematical Society 1446-7887/03 \$A2.00+0.00 
Here we denote by $U(\mathfrak{g})^{G}$ the algebra of invariants in the universal enveloping algebra $U(\mathfrak{g})$ with respect to the adjoint action of $G$. We have a similar equality $\sigma\left(U(\mathfrak{g})^{G}\right)=$ $\sigma\left(U\left(\mathfrak{g}^{\prime}\right)^{G^{\prime}}\right)$ also for a reductive dual pair $\left(G, G^{\prime}\right)$ in the complex orthogonal group $O_{N}$ and the spin representation $\sigma$. These correspondences between the two universal enveloping algebras have been investigated mainly in terms of eigenvalues of the central elements (see for example [9] or [6]). The aim of this paper is to describe these correspondences explicitly in terms of the bases of the Lie algebras. The main results are simple formulas for the generators of the algebras $U(\mathfrak{g})^{G}$ and $U\left(\mathfrak{g}^{\prime}\right)^{G^{\prime}}$ known by name of the 'Gelfand invariants.'

These generators given by I. M. Gelfand are defined for the classical groups as follows. First we consider the case of the general linear group $G L_{N}$. Let $E_{i j}$ be the standard basis of the Lie algebra $\mathfrak{g l}_{N}$, and form the matrix $\boldsymbol{E}=\boldsymbol{E}_{\mathfrak{g i}_{N}}=\left(E_{i j}\right)_{1 \leq i, j \leq N}$. We consider the trace of the power of this matrix:

$$
\operatorname{tr}\left(\boldsymbol{E}^{r}\right)=\sum_{1 \leq i_{1}, \ldots, i_{r} \leq N} E_{i_{1} i_{2}} E_{i_{2} i_{3}} \cdots E_{i_{r} i_{1}} .
$$

This element of the universal enveloping algebra is known to be invariant under the adjoint action of $G L_{N}$. Moreover the set $\left\{\operatorname{tr}\left(E^{r}\right) \mid 0 \leq r \leq N\right\}$ generates the algebra $U\left(\mathfrak{g l}_{N}\right)^{G L_{N}}$. We call these $\operatorname{tr}\left(\boldsymbol{E}^{r}\right)$ 's the 'Gelfand invariants' for $G L_{N}$ ([3], see also $[13,23])$.

Similar central elements of the universal enveloping algebra can be introduced for the Lie groups $G=O_{N}, S p_{N}$. Let $\Phi$ be the symmetric or alternating matrix associated with the bilinear form defining $G$. The Lie algebra $\mathfrak{g}=\operatorname{Lie}(G)$ is spanned by the elements $F_{i j}=E_{i j}-\Phi^{-1} E_{j i} \Phi$. From these generators, we arrange the matrix $\boldsymbol{F}=\boldsymbol{F}_{\mathfrak{g}}=\left(F_{i j}\right)_{1 \leq i, j \leq N}$. Then the trace of the power, $\operatorname{tr}\left(\boldsymbol{F}^{r}\right)$ is again invariant under the adjoint action of $G$. Moreover, the $\operatorname{set}\left\{\operatorname{tr}\left(F^{2 r}\right) \mid 0 \leq 2 r \leq N\right\}$ generates the algebra $U(\mathfrak{g})^{G}$. We also call these $\operatorname{tr}\left(\boldsymbol{F}^{r}\right)$ 's the 'Gelfand invariants' for $G([3,23])$.

REMARK. The algebra $U(\mathfrak{g})^{G}$ is obviously included in the center of $U(\mathfrak{g})$. This inclusion is actually an equality in the cases of $G=G L_{N}, S p_{N}$, and $O_{2 k+1}$. Only in the case of $G=O_{2 k}$, the algebra $U(\mathfrak{g})^{G}$ is a proper subset of the center.

Let us state the main theorems. Every reductive dual pair in the complex symplectic groups is written as a direct product of irreducible ones, which are classified into the two series $\left(G, G^{\prime}\right)=\left(G L_{m}, G L_{n}\right)$ and $\left(O_{m}, S p_{n}\right)([4,14,20])$. For each of these two types of irreducible dual pair, we can describe the correspondence between $U(\mathfrak{g})^{G}$ and $U\left(\mathfrak{g}^{\prime}\right)^{G^{\prime}}$ under the oscillator representation $\omega$ as follows:

THEOREM A. For the dual pair $\left(G L_{m}, G L_{n}\right)$ in $S p_{2 m n}$, the following equality holds:

$$
\begin{aligned}
& \omega\left(\operatorname{tr}\left(\left(\boldsymbol{E}-(n / 2) I_{m}\right)\left(\boldsymbol{E}-(n / 2+m) I_{m}\right)^{r}\right)\right) \\
& \quad=\omega\left(\operatorname{tr}\left(\left(\boldsymbol{E}^{\prime}-(m / 2) I_{n}\right)\left(\boldsymbol{E}^{\prime}-(m / 2+n) I_{n}\right)^{r}\right)\right) .
\end{aligned}
$$


Here we put $\boldsymbol{E}=E_{\mathfrak{g l}_{m}}$ and $E^{\prime}=E_{\mathfrak{g} \mathfrak{l}_{n}}$.

THEOREM B. For the dual pair $\left(O_{m}, S p_{n}\right)$ in $S p_{m n}$, the following equality holds:

$$
\begin{aligned}
& \omega\left(\operatorname{tr}\left(\left(\boldsymbol{F}-(n / 2) I_{m}\right)\left(\boldsymbol{F}-(n / 2+m-1 / 2) I_{m}\right)^{r}\right)\right) \\
& \quad=\omega\left(\operatorname{tr}\left(\left(\boldsymbol{F}^{\prime}-(m / 2) I_{n}\right)\left(\boldsymbol{F}^{\prime}-(m / 2+n+1 / 2) I_{n}\right)^{r}\right)\right) .
\end{aligned}
$$

Here we put $\boldsymbol{F}=\boldsymbol{F}_{\mathrm{o}_{m}}$ and $\boldsymbol{F}^{\prime}=\boldsymbol{F}_{\mathrm{sp}_{n}}$.

REMARK. As is seen from these theorems, the images of the Gelfand invariants for $G$ are written as linear sums of the images of the Gelfand invariants for $G^{\prime}$. This fact was first found by Klink, Leung, and Ton-That. However they did not describe this correspondence explicitly except for the Gelfand invariants of low degree. See [10] for the case of $\left(G L_{m}, G L_{n}\right)$, and $[12,11]$ for the case of $\left(O_{m}, S p_{n}\right)$.

Also for the reductive dual pairs in the complex orthogonal groups, we can similarly describe the correspondence between $U(\mathfrak{g})^{G}$ and $U\left(\mathfrak{g}^{\prime}\right)^{G^{\prime}}$ under the spin representation $\sigma$. The irreducible reductive dual pairs in the complex orthogonal groups are classified into the three series $([14,20]):\left(G L_{m}, G L_{n}\right),\left(O_{m}, O_{n}\right)$, and $\left(S p_{m}, S p_{n}\right)$.

THEOREM C. For the dual pair $\left(G L_{m}, G L_{n}\right)$ in $O_{2 m n}$, the following equality holds:

$$
\begin{aligned}
& \sigma\left(\operatorname{tr}\left(\left(E+(n / 2) I_{m}\right)\left(E+(n / 2-m) I_{m}\right)^{r}\right)\right) \\
& \quad=(-)^{r} \sigma\left(\operatorname{tr}\left(\left(E^{\prime}+(m / 2) I_{n}\right)\left(E^{\prime}+(m / 2-n) I_{n}\right)^{r}\right)\right) .
\end{aligned}
$$

Here we put $\boldsymbol{E}=\boldsymbol{E}_{\mathfrak{g} l_{m}}$ and $\boldsymbol{E}^{\prime}=\boldsymbol{E}_{\mathfrak{g} \mathfrak{l}_{n}}$.

THEOREM D. For the dual pair $\left(O_{m}, O_{n}\right)$ in $O_{m n}$, the following equality holds:

$$
\begin{aligned}
& \sigma\left(\operatorname{tr}\left(\left(\boldsymbol{F}+(n / 2) I_{m}\right)\left(\boldsymbol{F}+(n / 2-m+1 / 2) I_{m}\right)^{r}\right)\right) \\
& \quad=(-)^{r} \sigma\left(\operatorname{tr}\left(\left(\boldsymbol{F}^{\prime}+(m / 2) I_{n}\right)\left(\boldsymbol{F}^{\prime}+(m / 2-n+1 / 2) I_{n}\right)^{r}\right)\right) .
\end{aligned}
$$

Here we put $\boldsymbol{F}=\boldsymbol{F}_{\mathrm{o}_{m}}$ and $\boldsymbol{F}^{\prime}=\boldsymbol{F}_{\mathrm{o}_{n}}$.

THEOREM E. For the dual pair $\left(S p_{m}, S p_{n}\right)$ in $O_{m n}$, the following equality holds:

$$
\begin{aligned}
& \sigma\left(\operatorname{tr}\left(\left(\boldsymbol{F}+(n / 2) I_{m}\right)\left(\boldsymbol{F}+(n / 2-m-1 / 2) I_{m}\right)^{r}\right)\right) \\
& \quad=(-)^{r} \sigma\left(\operatorname{tr}\left(\left(\boldsymbol{F}^{\prime}+(m / 2) I_{n}\right)\left(\boldsymbol{F}^{\prime}+(m / 2-n-1 / 2) I_{n}\right)^{r}\right)\right) .
\end{aligned}
$$

Here we put $\boldsymbol{F}=F_{\mathbf{s p}_{\mathrm{m}}}$ and $\boldsymbol{F}^{\prime}=\boldsymbol{F}_{\mathbf{s p}_{\mathrm{n}}}$. 
We will verify these theorems by direct and consistent calculations under the realizations of the dual pairs in the Weyl algebra or the Clifford algebra.

For each of the dual pairs above, the decomposition of the oscillator representation or the spin representation into a direct sum of irreducible $G \times \mathfrak{g}^{\prime}$-modules has been investigated in detail $([9,6])$. Since the eigenvalues of the Gelfand invariants on the irreducible representations are given in [18] and [19] (see also [23]), some relation is expected between these results and our theorems. However it seems not so easy to deduce our theorems in fact from these irreducible decompositions.

We also comment on the relation with the Capelli type identities. The Capelli identity is a formula for the actions of the central elements of $U\left(\mathrm{gl}_{n}\right)$ named the 'Capelli elements', and gives another description of the correspondence between the centers of the universal enveloping algebras for the dual pair $\left(G L_{m}, G L_{n}\right)$ in $S p_{2 m n}$ $([1,2,21])$. The relation between the Capelli elements and the Gelfand invariants is given as an analogue of Newton's formula for the symmetric functions $([17,8,22])$. In particular, our Theorem $\mathrm{A}$ is also proved by combining the Capelli identity and this Newton type formula, while this proof cannot be applied to the other dual pairs.

Also in the case of the dual pair $\left(O_{m}, S p_{n}\right)$ in $S p_{m n}$, a description of this correspondence is given as an analogue of the Capelli identity in terms of eigenvalues in [16]. This is a consequence of the decomposition of the oscillator representation of $S p_{m n}$ into a direct sum of irreducible $O_{m} \times \mathfrak{s p}_{n}$-modules due to [9]. Connecting this result with the studies of the Sklyanin determinant in [15], we have an explicit description of the correspondence between $U\left(\mathfrak{o}_{m}\right)^{o_{m}}$ and $U\left(\mathfrak{s p}_{n}\right)^{S p_{n}}$ in terms of the bases of the Lie algebras. However, compared with our Theorem B, this description is somewhat complicated.

The author would like to express his sincere gratitude to Professor Tôru Umeda for the fruitful discussions and advice.

\section{The oscillator representation and the spin representation}

We first recall the realizations of the oscillator representation of the symplectic Lie algebras and the spin representation of the orthogonal Lie algebras. These two representations can be constructed in terms of the Weyl algebra and the Clifford algebra respectively in parallel ([5] and Appendix 2 of [6]).

1.1. The Weyl algebra and the oscillator representation. Let $W$ be a finitedimensional complex vector space with a symplectic form, that is, a non-degenerate alternating bilinear form $\langle\cdot, \cdot\rangle$. We define the Weyl algebra $\mathscr{W}(W)$ associated with $W$ by the quotient $\mathscr{W}(W)=T(W) / \mathscr{I}$, where $\mathscr{I}$ is the two-sided ideal of the tensor algebra $T(W)$ generated by the tensors $w_{1} \otimes w_{2}-w_{2} \otimes w_{1}-\left\langle w_{1}, w_{2}\right\rangle 1$ with $w_{1}, w_{2} \in W$. This $\mathscr{W}(W)$ is known to be a simple associative algebra. 
Let $V$ be a maximal isotropic subspace of $W$. Then the symmetric tensor algebra $S(V)$ has a structure of irreducible module of $\mathscr{W}(W)$. To see this, take another maximal isotropic subspace $V^{\dagger}$ such that $W=V \oplus V^{\dagger}$. This $V^{\dagger}$ is identified with the linear dual $V^{*}$ of $V$ via the symplectic form of $W$. For a vector $v \in V$ and a covector $v^{*} \in V^{*} \simeq V^{\dagger}$, we define the endomorphisms $M^{S}(v)$ and $D^{S}\left(v^{*}\right)$ on $S(V)$ by

$$
\begin{aligned}
M^{s}(v): v_{1} \cdots v_{k} & \mapsto v \cdot v_{1} \cdots v_{k}, \\
D^{s}\left(v^{*}\right): v_{1} \cdots v_{k} & \mapsto \sum_{i=1}^{k}\left\langle v^{*}, v_{i}\right\rangle \cdot v_{1} \cdots \widehat{v}_{i} \cdots v_{k},
\end{aligned}
$$

and call these the multiplication by $v$ and the derivation by $v^{*}$ respectively. Here $v_{i}$ 's are elements of $V$, and the symbol $\widehat{v}_{i}$ means that $v_{i}$ is omitted. Then the correspondence $W \ni v+v^{*} \mapsto M^{S}(v)+D^{S}\left(v^{*}\right)$ defines an isomorphism from $\mathscr{W}(W)$ into $\operatorname{End}(S(V))$. In particular, the Weyl algebra $\mathscr{W}(W)$ is isomorphic to the subalgebra $\operatorname{End}^{\circ}(S(V))$ of $\operatorname{End}(S(V))$ generated by the multiplications and the derivations on $S(V)$. Note that this $\operatorname{End}^{\circ}(S(V))$ is equal to the algebra of polynomial-coefficient differential operators under the natural identification of $S(V)$ with the space of polynomial functions on $V^{*}$.

The natural action of $S p(W)$ on $W$ extends to an action on $\mathscr{W}(W)$ by automorphisms. This action is infinitesimally inner in the following sense. Let $\mathscr{W}^{2}(W)$ be the subspace of $\mathscr{W}(W)$ spanned by the anti-commutators of elements in $W$ :

$$
w_{1} w_{2}+w_{2} w_{1}, \quad w_{1}, w_{2} \in W
$$

Then, with the commutator product, this $\mathscr{W}^{2}(W)$ is a Lie algebra isomorphic to $\operatorname{sp}(W)$. Moreover, the action of $\mathscr{W}^{2}(W)$ on $\mathscr{W}(W)$ defined by commutator is equal to the infinitesimal version of the natural action of $S p(W)$ on $\mathscr{W}(W)$. In particular, the natural action of $S p(W)$ on $\mathscr{W}^{2}(W)$ can be identified with the adjoint action on $\mathfrak{s p}(W)$. We call this $\mathscr{W}^{2}(W)$ the 'oscillator representation' of $\mathfrak{s p}(W)$.

1.2. The Clifford algebra and the spin representation. Let $W$ be a finitedimensional complex vector space with a non-degenerate symmetric bilinear form $\langle\cdot, \cdot\rangle$. We define the Clifford algebra $\mathscr{C}(W)$ associated with $W$ by the quotient $\mathscr{C}(W)=T(W) / \mathscr{J}$, where $\mathscr{J}$ is the two-sided ideal of the tensor algebra $T(W)$ generated by the tensors $w_{1} \otimes w_{2}+w_{2} \otimes w_{1}-\left\langle w_{1}, w_{2}\right\rangle 1$ with $w_{1}, w_{2} \in W$.

As in the case of the Weyl algebra, the Lie algebra $\mathfrak{o}(W)$ is naturally embedded into the Clifford algebra $\mathscr{C}(W)$. Let $\mathscr{C}^{2}(W)$ be the subspace of $\mathscr{C}(W)$ spanned by the commutators of elements in $W$ :

$$
w_{1} w_{2}-w_{2} w_{1}, \quad w_{1}, w_{2} \in W .
$$

Then the commutator product makes this $\mathscr{C}^{2}(W)$ into a Lie algebra isomorphic to $o(W)$, and the action of $\mathscr{C}^{2}(W)$ on $\mathscr{C}(W)$ defined by commutator is identified 
with the infinitesimal version of the natural action of $O(W)$ on $\mathscr{C}(W)$. In particular, the natural action of $O(W)$ on $\mathscr{C}^{2}(W)$ coincides with the adjoint action on $O(W)$. We call this $\mathscr{C}^{2}(W)$ the 'spin representation' of $o(W)$.

This $\mathscr{C}^{2}(W)$ exponentiates to a two-fold cover of $S O(W)$, which is called the 'spin group.' The natural action of $S O(W)$ on $\mathscr{C}(W)$ is realized as the action of this spin group by conjugation.

When the dimension of $W$ is even, the Clifford algebra $\mathscr{C}(W)$ is a simple algebra, and its irreducible module is realized as the exterior algebra of a maximal isotropic subspace of $W$. Let $V$ and $V^{\dagger}$ be isotropic subspaces of $W$ such that $W=V \oplus V^{\dagger}$. Via the bilinear form of $W$, we identify the space $V^{\dagger}$ with the linear dual $V^{*}$ of $V$. For a vector $v \in V$ and a covector $v^{*} \in V^{*} \simeq V^{\dagger}$, we define the multiplication $M^{\wedge}(v)$ and the derivation $D^{\Lambda}\left(v^{*}\right)$ on $\Lambda(V)$ by

$$
\begin{aligned}
M^{\wedge}(v): v_{1} \cdots v_{k} & \mapsto v \cdot v_{1} \cdots v_{k}, \\
D^{\wedge}\left(v^{*}\right): v_{1} \cdots v_{k} & \mapsto \sum_{i=1}^{k}(-)^{i-1}\left\langle v^{*}, v_{i}\right\rangle \cdot v_{1} \cdots \widehat{v}_{i} \cdots v_{k}
\end{aligned}
$$

with $v_{1}, \ldots, v_{k} \in V$. Then the correspondence $W \ni v+v^{*} \mapsto M^{\Lambda}(v)+D^{\Lambda}\left(v^{*}\right)$ defines an isomorphism from $\mathscr{C}(W)$ to the algebra $\operatorname{End}^{\circ}(\Lambda(V))$ generated by the multiplications and the derivations on $\Lambda(V)$. This $\operatorname{End}^{\circ}(\Lambda(V))$ is actually equal to $\operatorname{End}(\Lambda(V))$.

REMARK. When the dimension of $W$ is odd, the Clifford algebra $\mathscr{C}(W)$ is equivalent to the direct sum of two matrix algebras of dimension $\operatorname{dim} \mathscr{C}(W) / 2$.

\section{The pair $\left(G L_{m}, G L_{n}\right)$ in $S p_{2 m n}$}

The irreducible reductive dual pairs in the symplectic groups fall into the two classes, type I and type II, namely the pair $\left(O_{m}, S p_{n}\right)$ in $S p_{m n}$ and the pair $\left(G L_{m}, G L_{n}\right)$ in $S p_{2 m n}([4,14,20])$. First in this section, we study the latter pair.

For two finite-dimensional vector spaces $V_{1}$ and $V_{2}$, the groups $G L\left(V_{1}\right)$ and $G L\left(V_{2}\right)$ constitute a dual pair as the subgroups of $G L\left(V_{1} \otimes V_{2}\right)$. This pair can also be regarded as a dual pair in a larger symplectic group as follows. Put $V=V_{1} \otimes V_{2}$, and let $V^{*}$ be its linear dual. We define a symplectic form $\langle\cdot, \cdot\rangle$ on $V \oplus V^{*}$ by

$$
\left\langle u+u^{*}, v+v^{*}\right\rangle=u^{*}(v)-v^{*}(u), \quad u, v \in V, \quad u^{*}, v^{*} \in V^{*},
$$

so that $V$ and $V^{*}$ are maximal isotropic subspaces. The group $G L(V)$ acts on this space $V \oplus V^{*}$ isometrically by $g \cdot\left(u, u^{*}\right)=\left(g u,{ }^{t} g^{-1} u^{*}\right)$ with $g \in G L(V)$ and $\left(u, u^{*}\right) \in V \oplus V^{*}$. By this action we regard $G L(V)$ as the subgroup of $\operatorname{Sp}\left(V \oplus V^{*}\right)$. 
The subgroups $G L\left(V_{1}\right)$ and $G L\left(V_{2}\right)$ of $G L(V)$ still constitute a dual pair as the subgroups of $S p\left(V \oplus V^{*}\right)$.

For the oscillator representation $\omega$ of $\operatorname{sp}\left(V \oplus V^{*}\right)$, the following remarkable fact is known: the invariants under the natural actions of $G L\left(V_{1}\right)$ and $G L\left(V_{2}\right)$ on $\omega\left(U\left(\mathfrak{s p}\left(V \oplus V^{*}\right)\right)\right)$ are generated by $\omega\left(\mathfrak{g l}\left(V_{2}\right)\right)$ and $\omega\left(\mathfrak{g l}\left(V_{1}\right)\right)$ respectively ([5]). In particular, we see that the images of $U\left(\mathfrak{g l}\left(V_{1}\right)\right)^{G L\left(V_{1}\right)}$ and $U\left(\mathfrak{g l}\left(V_{2}\right)\right)^{G L\left(V_{2}\right)}$ under $\omega$ coincide.

The first theorem of this paper is the following relation, which explicitly describes this correspondence with the Gelfand invariants. Let $E_{i j}$ and $E_{i j}^{\prime}$ be the standard bases of $\mathfrak{g l}\left(V_{1}\right)$ and $\mathfrak{g l}\left(V_{2}\right)$ respectively, and consider the matrices $\boldsymbol{E}=\left(E_{i j}\right)_{1 \leq i, j \leq m}$ and $E^{\prime}=\left(E_{i j}^{\prime}\right)_{1 \leq i, j \leq n}$. Here we put $m=\operatorname{dim} V_{1}$ and $n=\operatorname{dim} V_{2}$.

THEOREM 2.1. The following equality holds:

$$
\begin{aligned}
& \omega\left(\operatorname{tr}\left(\left(\boldsymbol{E}-(n / 2) I_{m}\right)\left(\boldsymbol{E}-(n / 2+m) I_{m}\right)^{r}\right)\right) \\
& \quad=\omega\left(\operatorname{tr}\left(\left(\boldsymbol{E}^{\prime}-(m / 2) I_{n}\right)\left(\boldsymbol{E}^{\prime}-(m / 2+n) I_{n}\right)^{r}\right)\right) .
\end{aligned}
$$

In the remainder of this section, we prove Theorem 2.1. First we describe the actions of $\mathfrak{g l}\left(V_{1}\right)$ and $\mathfrak{g l}\left(V_{2}\right)$ in terms of the Weyl algebra. Let $v_{i j}$ be the standard basis of $V=V_{1} \otimes V_{2}$ and $v_{i j}^{*}$ its dual basis. Then the multiplications $x_{i j}=M^{s}\left(v_{i j}\right)$ and the derivations $x_{i j}^{*}=D^{S}\left(v_{i j}^{*}\right)$ on $S(V)$ satisfy the following relations (the canonical commutation relations):

$$
\left[x_{i j}, x_{k l}\right]=\left[x_{i j}^{*}, x_{k l}^{*}\right]=0, \quad\left[x_{i j}^{*}, x_{k l}\right]=\delta_{i k} \delta_{j l} .
$$

Here the brackets are used to denote commutator: $[a, b]=a b-b a$. As seen in Section 1, these $x_{i j}$ 's and $x_{i j}^{*}$ 's generate the Weyl algebra $\mathscr{W}\left(V \oplus V^{*}\right) \simeq \operatorname{End}^{\circ}(S(V))$. With this notation the actions of the two Lie algebras $\mathfrak{g l}\left(V_{1}\right)$ and $\mathfrak{g l}\left(V_{2}\right)$ are given as follows ([6]):

$$
\omega\left(E_{i j}\right)=\sum_{s=1}^{n} x_{i s} x_{j s}^{*}+\frac{n}{2} \delta_{i j}, \quad \omega\left(E_{i j}^{\prime}\right)=\sum_{s=1}^{m} x_{s i} x_{s j}^{*}+\frac{m}{2} \delta_{i j} .
$$

These are simply written as $\omega(E)=X^{\prime} X^{*}+(n / 2) I_{m}$ and $\omega\left(E^{\prime}\right)={ }^{t} X X^{*}+(m / 2) I_{n}$ by introducing two $m \times n$ matrices $X=\left(x_{i j}\right)$ and $X^{*}=\left(x_{i j}^{*}\right)$. In particular, Theorem 2.1 is rewritten as

$$
\operatorname{tr}\left(X^{\prime} X^{*}\left(X^{t} X^{*}-m I_{m}\right)^{r}\right)=\operatorname{tr}\left({ }^{t} X X^{*}\left({ }^{t} X X^{*}-n I_{n}\right)^{r}\right)
$$

Let us deduce this equality using the canonical commutation relations (2.1). To do this systematically, we employ the following three lemmas: 
LEMMA 2.2. The following equalities hold:

$$
{ }^{t} X^{*} X-m I_{n}={ }^{t}\left({ }^{t} X X^{*}\right), \quad X^{* t} X-n I_{m}={ }^{t}\left(X^{t} X^{*}\right) .
$$

LEMMA 2.3. The entries of $X^{\prime} X^{*}$ and $X X^{*}$ mutually commute:

$$
\left[\left(X^{t} X^{*}\right)_{i j},\left({ }^{t} X X^{*}\right)_{k l}\right]=0 .
$$

LEMMA 2.4. The following equality holds: $\left(X^{\prime}\left({ }^{\prime} X X^{*}\right)\right)_{i j}=\sum_{s=1}^{m} x_{s j}\left(X^{\prime} X^{*}\right)_{i s}$.

The proofs of these lemmas are all easy. In fact Lemma 2.2 is immediate from the relation $\left[x_{i j}^{*}, x_{k l}\right]=\delta_{i k} \delta_{j l}$ in (2.1). Lemma 2.3 is a direct consequence of the fact that the actions of the two Lie algebras $\mathfrak{g l}\left(V_{1}\right)$ and $\mathfrak{g l}\left(V_{2}\right)$ are mutually commutative. Lemma 2.4 can be also checked by noting that the entries of $X$ commute with each other.

Our task is now to prove

$$
\operatorname{tr}\left(X^{t}\left({ }^{t} X X^{*}\right)^{r} \cdot{ }^{t} X^{*}\right)=\operatorname{tr}\left({ }^{t} X^{t}\left(X^{t} X^{*}\right)^{r} X^{*}\right),
$$

because Lemma 2.2 implies the equalities

$$
\begin{aligned}
X^{t} X^{*}\left(X^{t} X^{*}-m I_{m}\right)^{r} & =X\left({ }^{t} X^{*} X-m I_{n}\right)^{r} \cdot{ }^{t} X^{*}=X^{t}\left({ }^{t} X X^{*}\right)^{r} \cdot{ }^{t} X^{*}, \\
{ }^{t} X X^{*}\left({ }^{t} X X^{*}-n I_{n}\right)^{r} & ={ }^{t} X\left(X^{* t} X-n I_{m}\right)^{r} X^{*}={ }^{t} X^{t}\left(X^{t} X^{*}\right)^{r} X^{*} .
\end{aligned}
$$

Here the notation ' $Z$ r indicates the $r$ th power of the transpose of a matrix $Z$. To verify this (2.3), we claim the following relation:

PROPOSITION 2.5. For any $Z \in \operatorname{Mat}_{m, n}\left(\mathscr{W}\left(V \oplus V^{*}\right)\right)$, the following equality holds:

$$
\operatorname{tr}\left(X^{t}\left({ }^{t} X X^{*}\right)^{r} \cdot{ }^{t} Z\right)=\operatorname{tr}\left(X^{t}\left({ }^{t} X X^{*}\right)^{r-1} \cdot{ }^{t}\left({ }^{t}\left(X^{t} X^{*}\right) Z\right)\right) .
$$

PROOF. By a straightforward calculation and Lemma 2.4, we have

$$
\begin{aligned}
\operatorname{tr}\left(X^{\prime}\left({ }^{t} X X^{*}\right)^{r} \cdot{ }^{t} Z\right) & =\operatorname{tr}\left(X^{t}\left({ }^{t} X X^{*}\right){ }^{t}\left({ }^{t} X X^{*}\right)^{r-1} \cdot{ }^{t} Z\right) \\
& =\sum_{i, j, k}\left(X^{t}\left({ }^{t} X X^{*}\right)\right)_{i j}\left({ }^{t}\left({ }^{t} X X^{*}\right)^{r-1}\right)_{j k}\left({ }^{t} Z\right)_{k i} \\
& =\sum_{i, j, k, s} x_{s j}\left(X^{t} X^{*}\right)_{i s}\left({ }^{t}\left({ }^{t} X X^{*}\right)^{r-1}\right)_{j k}\left({ }^{t} Z\right)_{k i} .
\end{aligned}
$$

Since $\left(X^{t} X^{*}\right)_{i s}$ and $\left({ }^{t}\left({ }^{\prime} X X^{*}\right)^{r-1}\right)_{j k}$ are commutative by Lemma 2.3 , this is equal to

$$
\sum_{i, j, k, s} x_{s j}\left({ }^{t}\left({ }^{\prime} X X^{*}\right)^{r-1}\right)_{j k}\left(X^{t} X^{*}\right)_{i s}\left({ }^{t} Z\right)_{k i}
$$

Moreover this is equal to the right-hand side of our assertion. 
Since the matrix $Z$ is arbitrary, we can use the relation in Proposition 2.5 repeatedly. Thus we arrive at the following identity:

COROLlARY 2.6. For any $Z \in \operatorname{Mat}_{m, n}\left(\mathscr{W}\left(V \oplus V^{*}\right)\right)$, the following equality holds:

$$
\operatorname{tr}\left(X^{t}\left({ }^{t} X X^{*}\right)^{r} \cdot{ }^{\prime} Z\right)=\operatorname{tr}\left(X \cdot{ }^{t}\left({ }^{\prime}\left(X^{\prime} X^{*}\right)^{r} Z\right)\right)=\operatorname{tr}\left({ }^{t} X^{t}\left(X^{t} X^{*}\right)^{r} Z\right) .
$$

Now Theorem 2.1 is almost proved. Indeed our goal (2.3) is obtained by replacing $Z$ by $X^{*}$ in the equality of Corollary 2.6 .

\section{The pair $\left(O_{m}, S p_{n}\right)$ in $S p_{m n}$}

In this section, we study the dual pairs of type I in the symplectic groups, namely the pair $\left(O_{m}, S p_{n}\right)$ in $S p_{m n}$.

To introduce this pair, we start with two finite-dimensional vector spaces $W_{1}$ and $W_{2}$ equipped with non-degenerate bilinear forms. We assume that the bilinear form $\langle\cdot, \cdot\rangle_{1}$ of $W_{1}$ is symmetric, and the bilinear form $\langle\cdot, \cdot\rangle_{2}$ of $W_{2}$ is alternating. From these bilinear forms, we define a symplectic form $\langle\cdot, \cdot\rangle$ on the tensor product $W=W_{1} \otimes W_{2}$ by $\left\langle u_{1} \otimes u_{2}, v_{1} \otimes v_{2}\right\rangle=\left\langle u_{1}, v_{1}\right\rangle_{1} \cdot\left\langle u_{2}, v_{2}\right\rangle_{2}$, and consider the isometry group $S p(W)$. Then the isometry groups $O\left(W_{1}\right)$ and $S p\left(W_{2}\right)$ constitute a dual pair as the subgroups of $S p(W)$. Thus we have the dual pair $\left(O_{m}, S p_{n}\right)$ in $S p_{m n}$ with $m=\operatorname{dim} W_{1}$ and $n=\operatorname{dim} W_{2}$.

For this dual pair and the oscillator representation $\omega$ of $s p_{m n}$, the following fact is known: the invariants under the actions $O_{m}$ and $S p_{n}$ on $\omega\left(U\left(s \mathfrak{p}_{m n}\right)\right)$ are gener-

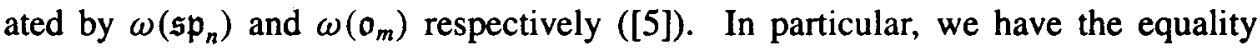
$\omega\left(U\left(\mathfrak{o}_{m}\right)^{O_{m}}\right)=\omega\left(U\left(\mathfrak{s p _ { n }}\right)^{S p_{n}}\right)$.

As a description of this correspondence between the universal enveloping algebras, we have the following relation. We put $\boldsymbol{F}=\boldsymbol{F}_{\mathrm{o}_{m}}$ and $\boldsymbol{F}^{\prime}=\boldsymbol{F}_{\mathbf{s p}_{\mathrm{n}}}$.

THEOREM 3.1. The follawing equality holds:

$$
\begin{aligned}
& \omega\left(\operatorname{tr}\left(\left(\boldsymbol{F}-(n / 2) I_{m}\right)\left(\boldsymbol{F}-(n / 2+m-1 / 2) I_{m}\right)^{r}\right)\right) \\
& \quad=\omega\left(\operatorname{tr}\left(\left(\boldsymbol{F}^{\prime}-(m / 2) I_{n}\right)\left(\boldsymbol{F}^{\prime}-(m / 2+n+1 / 2) I_{n}\right)^{r}\right)\right) .
\end{aligned}
$$

To prove Theorem 3.1, we first describe the actions of the Lie algebras $\mathfrak{o}_{m}$ and $\mathfrak{s p} \mathfrak{p}_{n}$ under the oscillator representation $\omega$ in terms of the Weyl algebra $\mathscr{W}(W)$. Denote by $\Phi=\left(\Phi_{i j}\right)$ and $\Psi=\left(\Psi_{i j}\right)$ the matrices associated with the bilinear forms $\langle\cdot, \cdot\rangle_{1}$ and $\langle\cdot, \cdot\rangle_{2}$, so that we have $\left\langle p_{i j}, p_{k l}\right\rangle=\Phi_{i k} \Psi_{j l}$ for the canonical basis $p_{i j}$ of $W=W_{1} \otimes W_{2}$. In particular, these $p_{i j}$ 's satisfy the commutation relation $\left[p_{i j}, p_{k l}\right]=\left\langle p_{i j}, p_{k l}\right\rangle=$ $\Phi_{i k} \Psi_{j l}$ as the elements of the Weyl algebra $\mathscr{W}(W)$. Form the $m \times n$ matrices $P=\left(p_{i j}\right)$ 
and $P^{*}=\left(p_{i j}^{*}\right)=\Phi^{-1} P^{*} \Psi^{-1}$, so that the commutation relation $\left[p_{i j}^{*}, p_{k l}\right]=\delta_{i k} \delta_{j l}$ holds. Then the actions of $\mathfrak{o}_{m}$ and $\mathfrak{s p}_{n}$ are given as follows ([6]):

$$
\begin{aligned}
\omega(\boldsymbol{F}) & =P \Psi^{-1}{ }^{t} P^{t} \Phi^{-1}+(n / 2) I_{m}=P^{t} P^{*}+(n / 2) I_{m}, \\
\omega\left(F^{\prime}\right) & ={ }^{t} P \Phi^{-1} P^{t} \Psi^{-1}+(m / 2) I_{n}={ }^{t} P P^{*}+(m / 2) I_{n} .
\end{aligned}
$$

Thus, for Theorem 3.1, it suffices to prove the relation

$$
\operatorname{tr}\left(P^{t} P^{*}\left(P^{t} P^{*}-(m-1 / 2) I_{m}\right)^{r}\right)=\operatorname{tr}\left({ }^{t} P P^{*}\left({ }^{t} P P^{*}-(n+1 / 2) I_{n}\right)^{r}\right) .
$$

We deduce this equality using the following three lemmas it a way similar to the proof of Theorem 2.1:

LEMMA 3.2. The following equalities hold:

$$
{ }^{t} P^{*} P-m I_{n}={ }^{t}\left(P P^{*}\right), \quad P^{* t} P-n I_{m}={ }^{t}\left(P^{t} P^{*}\right) .
$$

Lemma 3.3. The entries of $P^{t} P^{*}$ and ${ }^{t} P P^{*}$ mutually commute:

$$
\left[\left(P^{t} P^{*}\right)_{i j},\left({ }^{t} P P^{*}\right)_{k l}\right]=0 .
$$

LEMMA 3.4. The following equality holds:

$$
\left(P^{t}\left({ }^{t} P P^{*}+(1 / 2) I_{n}\right)\right)_{i j}=\sum_{s=1}^{m} p_{s j}\left(P^{t} P^{*}-(1 / 2) I_{m}\right)_{i s} .
$$

The proofs of these lemmas are almost the same as those of Lemmas 2.2-2.4. Indeed Lemma 3.2 is a consequence of the commutation relation $\left[p_{i j}^{*}, p_{k l}\right]=\delta_{i k} \delta_{j l}$, and Lemma 3.3 is immediate from the fact that the two actions of $O_{m}$ and $S p_{n}$ are commutative each other. Lemma 3.4 is a bit more complicated than Lemma 2.4, but is also seen by a direct calculation from the commutation relations of $p_{i j}$ 's.

By using Lemma 3.2, our goal (3.1) is now rewritten as

$$
\operatorname{tr}\left(P^{t}\left({ }^{t} P P^{*}+\frac{1}{2} I_{n}\right)^{r} \cdot{ }^{t} P^{*}\right)=\operatorname{tr}\left({ }^{t} P^{t}\left(P^{t} P^{*}-\frac{1}{2} I_{m}\right)^{r} P^{*}\right) .
$$

To show this, we claim the following proposition. This is seen from Lemma 3.3 and Lemma 3.4 in a way similar to the proof of Proposition 2.5.

PROPOSITION 3.5. For any $Z \in \operatorname{Mat}_{m, n}(\mathscr{W}(W))$, the following equality holds:

$$
\operatorname{tr}\left(P^{t}\left({ }^{t} P P^{*}+\frac{1}{2} I_{n}\right)^{r} \cdot{ }^{t} Z\right)=\operatorname{tr}\left(P^{t}\left({ }^{t} P P^{*}+\frac{1}{2} I_{n}\right)^{r-1} \cdot{ }^{t}\left({ }^{t}\left(P^{t} P^{*}-\frac{1}{2} I_{m}\right) Z\right)\right) .
$$


Repeating this relation $r$ times, we arrive at the following corollary. Our goal (3.2) is obtained by replacing $Z$ by $P^{*}$ in this equality.

COROLLARY 3.6. For any $Z \in \operatorname{Mat}_{m, n}(\mathscr{W}(W))$, the following equality holds:

$$
\begin{aligned}
\operatorname{tr}\left(P^{\prime}\left({ }^{t} P P^{*}+\frac{1}{2} I_{n}\right)^{r} \cdot{ }^{t} Z\right) & =\operatorname{tr}\left(P \cdot{ }^{t}\left({ }^{t}\left(P^{t} P^{*}-\frac{1}{2} I_{m}\right)^{r} Z\right)\right) \\
& =\operatorname{tr}\left({ }^{t} P^{t}\left(P^{t} P^{*}-\frac{1}{2} I_{m}\right)^{r} Z\right) .
\end{aligned}
$$

\section{The pair $\left(G L_{m}, G L_{n}\right)$ in $O_{2 m n}$}

As in the case of the symplectic group, the irreducible reductive dual pairs in the orthogonal groups fall into the two classes, type I and type II $([14,20])$. In this section, we study the dual pairs of type II in the orthogonal groups, namely the pair $\left(G L_{m}, G L_{n}\right)$ in $O_{2 m n}$.

The dual pair $\left(G L\left(V_{1}\right), G L\left(V_{2}\right)\right)$ in $G L\left(V_{1} \otimes V_{2}\right)$, which we considered in Section 2, can be also regarded as a dual pair in a larger orthogonal group. Put $V=V_{1} \otimes V_{2}$, and denote its linear dual by $V^{*}$. We define a symmetric bilinear form on $V \oplus V^{*}$ by

$$
\left\langle u+u^{*}, v+v^{*}\right\rangle=u^{*}(v)+v^{*}(u), \quad u, v \in V, u^{*}, v^{*} \in V^{*} .
$$

The group $G L(V)$ acts on this space $V \oplus V^{*}$ isometrically by $g \cdot\left(u, u^{*}\right)=\left(g u, g^{-1} u^{*}\right)$ with $g \in G L(V)$ and $\left(u, u^{*}\right) \in V \oplus V^{*}$. By this action we regard $G L(V)$ as the subgroup of $O\left(V \oplus V^{*}\right)$. The pair $\left(G L\left(V_{1}\right), G L\left(V_{2}\right)\right)$ still constitutes a dual pair as the subgroups of $O\left(V \oplus V^{*}\right)$.

Let us consider the spin representation $\sigma$ of $o\left(V \oplus V^{*}\right)$ on $\Lambda(V)$. As in the case of the dual pairs in the symplectic group, it is known that the invariants of $G L\left(V_{1}\right)$ and $G L\left(V_{2}\right)$ on $\sigma\left(U\left(\mathfrak{o}\left(V \oplus V^{*}\right)\right)\right)$ are generated by $\sigma\left(\mathfrak{g l}\left(V_{2}\right)\right)$ and $\sigma\left(\mathfrak{g l}\left(V_{1}\right)\right)$ respectively ([5]). In particular, we see that the images of $U\left(\mathfrak{g l}\left(V_{1}\right)\right)^{G L\left(V_{1}\right)}$ and $U\left(\mathfrak{g l}\left(V_{2}\right)\right)^{G L\left(V_{2}\right)}$ under $\sigma$ coincide.

As an explicit description of this correspondence, we have the following relation similar to Theorem 2.1. Let $E_{i j}$ and $E_{i j}^{\prime}$ be the standard bases of $\mathfrak{g l}\left(V_{1}\right)$ and $\mathfrak{g l}\left(V_{2}\right)$ respectively, and consider the matrices $\boldsymbol{E}=\left(E_{i j}\right)_{1 \leq i, j \leq m}$ and $\boldsymbol{E}^{\prime}=\left(E_{i j}^{\prime}\right)_{1 \leq i, j \leq n}$. Here we put $m=\operatorname{dim} V_{1}$ and $n=\operatorname{dim} V_{2}$.

THEOREM 4.1. The following equality holds:

$$
\begin{aligned}
& \sigma\left(\operatorname{tr}\left(\left(E+(n / 2) I_{m}\right)\left(E+(n / 2-m) I_{m}\right)^{r}\right)\right) \\
& \quad=(-)^{r} \sigma\left(\operatorname{tr}\left(\left(E^{\prime}+(m / 2) I_{n}\right)\left(E^{\prime}+(m / 2-n) I_{n}\right)^{r}\right)\right)
\end{aligned}
$$


To prove Theorem 4.1, let us write down the actions of $\mathfrak{g l}\left(V_{1}\right)$ and $\mathfrak{g l}\left(V_{2}\right)$ in terms of the Clifford algebra. Let $v_{i j}$ be the standard basis of $V=V_{1} \otimes V_{2}$, and $v_{i j}^{*}$ its dual basis. Then the multiplication $y_{i j}=M^{\Lambda}\left(v_{i j}\right)$ and derivation $y_{i j}^{*}=D^{\Lambda}\left(v_{i j}^{*}\right)$ on $\Lambda(V)$ satisfy the following relations (the canonical anti-commutation relations):

$$
\left\{y_{i j}, y_{k l}\right\}=\left\{y_{i j}^{*}, y_{k l}^{*}\right\}=0, \quad\left\{y_{i j}^{*}, y_{k l}\right\}=\delta_{i k} \delta_{j l} .
$$

Here the braces are used to denote anti-commutator: $\{a, b\}=a b+b a$. As seen in Section 1, these $y_{i j}$ and $y_{i j}^{*}$ generate the Clifford algebra $\mathscr{C}\left(V \oplus V^{*}\right) \simeq \operatorname{End}^{\circ}(\Lambda(V))$. With this notation the actions of $\mathfrak{g l}\left(V_{1}\right)$ and $\mathfrak{g l}\left(V_{2}\right)$ are expressed as follows ([6]):

$$
\sigma\left(E_{i j}\right)=\sum_{s=1}^{n} y_{i s} y_{j s}^{*}-\frac{n}{2} \delta_{i j}, \quad \sigma\left(E_{i j}^{\prime}\right)=\sum_{s=1}^{m} y_{s i} y_{s j}^{*}-\frac{m}{2} \delta_{i j} .
$$

We introduce the two $m \times n$ matrices $Y=\left(y_{i j}\right)$ and $Y^{*}=\left(y_{i j}^{*}\right)$, so that we have $\sigma(E)=Y^{\prime} Y^{*}-(n / 2) I_{m}$ and $\sigma\left(E^{\prime}\right)={ }^{\prime} Y Y^{*}-(m / 2) I_{n}$. Then Theorem 4.1 is rewritten as

$$
\operatorname{tr}\left(Y^{t} Y^{*}\left(Y^{t} Y^{*}-m I_{m}\right)^{r}\right)=(-)^{r} \operatorname{tr}\left({ }^{t} Y Y^{*}\left({ }^{t} Y Y^{*}-n I_{n}\right)^{r}\right) .
$$

The proof of this equality is essentially the same as that of (2.3). Therefore we only state the counterparts of Lemmas 2.2-2.4, Proposition 2.5, and Corollary 2.6, and omit the details.

LEMMA 4.2. The following equalities hold:

$$
{ }^{t} Y^{*} Y-m I_{n}=-{ }^{t}\left({ }^{t} Y Y^{*}\right), \quad Y^{* t} Y-n I_{m}=-{ }^{t}\left(Y^{t} Y^{*}\right) .
$$

LEMMA 4.3. The entries of $Y^{t} Y^{*}$ and $Y Y^{*}$ mutually commute:

$$
\left[\left(Y^{t} Y^{*}\right)_{i j},\left({ }^{t} Y Y^{*}\right)_{k l}\right]=0 .
$$

LEMMA 4.4. The following equality holds: $\left(Y^{t}\left({ }^{\prime} Y Y^{*}\right)\right)_{i j}=-\sum_{s=1}^{m} y_{s j}\left(Y^{\prime} Y^{*}\right)_{i s}$.

PROPOSITION 4.5. For any $Z \in \operatorname{Mat}_{m, n}\left(\mathscr{C}\left(V \oplus V^{*}\right)\right)$, the following equality holds:

$$
\operatorname{tr}\left(Y^{t}\left({ }^{t} Y Y^{*}\right) \cdot{ }^{t} Z\right)=-\operatorname{tr}\left(Y^{t}\left({ }^{t} Y Y^{*}\right)^{r-1} \cdot{ }^{t}\left({ }^{t}\left(Y^{t} Y^{*}\right) Z\right)\right) .
$$

COROLLARY 4.6. For any $Z \in \operatorname{Mat}_{m, n}\left(\mathscr{C}\left(V \oplus V^{*}\right)\right)$, the following equality holds:

$$
\operatorname{tr}\left(Y^{\prime}\left({ }^{t} Y Y^{*}\right)^{r} \cdot{ }^{\prime} Z\right)=(-)^{r} \operatorname{tr}\left(Y \cdot{ }^{t}\left({ }^{t}\left(Y^{t} Y^{*}\right)^{r} Z\right)\right)=(-)^{r} \operatorname{tr}\left({ }^{t} Y^{t}\left(Y^{\prime} Y^{*}\right)^{r} Z\right) .
$$




\section{The pairs $\left(O_{m}, O_{n}\right)$ and $\left(S p_{m}, S p_{n}\right)$ in $O_{m n}$}

Finally we study the dual pairs of type $I$ in the orthogonal groups, namely the two pairs $\left(O_{m}, O_{n}\right)$ and $\left(S p_{m}, S p_{n}\right)$ in $O_{m n}$.

As in Section 3, we start with two finite-dimensional vector spaces $W_{1}$ and $W_{2}$ equipped with non-degenerate bilinear forms $\langle\cdot, \cdot\rangle_{1}$ and $\langle\cdot, \cdot\rangle_{2}$. We assume that both bilinear forms are simultaneously symmetric or alternating, that is, there exists a fixed $\varepsilon= \pm 1$ such that $\left\langle u_{1}, v_{1}\right\rangle_{1}=\varepsilon\left\langle v_{1}, u_{1}\right\rangle_{1}$ and $\left\langle u_{2}, v_{2}\right\rangle_{2}=\varepsilon\left\langle v_{2}, u_{2}\right\rangle_{2}$. We define a symmetric bilinear form $\langle\cdot, \cdot\rangle$ on $W=W_{1} \otimes W_{2}$ by

$$
\left\langle u_{1} \otimes u_{2}, v_{1} \otimes v_{2}\right\rangle=\left\langle u_{1}, v_{1}\right\rangle_{1} \cdot\left\langle u_{2}, v_{2}\right\rangle_{2},
$$

and consider the isometry group $O(W)$. Let $G$ and $G^{\prime}$ be the isometry groups of $W_{1}$ and $W_{2}$ respectively. These $G$ and $G^{\prime}$ constitute a dual pair as the subgroups of $O(W)$. Thus, according to $\varepsilon= \pm 1$, we have two types of dual pairs $\left(O_{m}, O_{n}\right)$ and $\left(S p_{m}, S p_{n}\right)$ in $O_{m n}$. Here we put $m=\operatorname{dim} W_{1}$ and $n=\operatorname{dim} W_{2}$.

For the spin representation $\sigma$ of $o_{m n}$, it is known that the invariants under the actions of $G$ and $G^{\prime}$ on $\sigma\left(U\left(\mathfrak{o}_{m n}\right)\right)$ are generated by the images of the Lie algebras $\mathfrak{g}^{\prime}=\operatorname{Lie}\left(G^{\prime}\right)$ and $\mathfrak{g}=\operatorname{Lie}(G)$ respectively ([5]). In particular, we have the equality $\sigma\left(U(\mathfrak{g})^{G}\right)=$ $\sigma\left(U\left(\mathfrak{g}^{\prime}\right)^{G^{\prime}}\right)$. As a description of this correspondence between the universal enveloping algebras, we have the following theorem. We put $\boldsymbol{F}=\boldsymbol{F}_{\mathfrak{g}}$ and $\boldsymbol{F}^{\prime}=\boldsymbol{F}_{\mathfrak{g}^{\prime}}$.

THEOREM 5.1. The following equality holds:

$$
\begin{aligned}
& \sigma\left(\operatorname{tr}\left(\left(\boldsymbol{F}+(n / 2) I_{m}\right)\left(\boldsymbol{F}+(n / 2-m+\varepsilon / 2) I_{m}\right)^{r}\right)\right) \\
& \quad=(-)^{r} \sigma\left(\operatorname{tr}\left(\left(\boldsymbol{F}^{\prime}+(m / 2) I_{n}\right)\left(\boldsymbol{F}^{\prime}+(m / 2-n+\varepsilon / 2) I_{n}\right)^{r}\right)\right) .
\end{aligned}
$$

Preparatory to proving Theorem 5.1, we describe the actions of the Lie algebras $\mathfrak{g}$ and $g^{\prime}$ in terms of the Clifford algebra $\mathscr{C}(W)$. We denote by $\Phi=\left(\Phi_{i j}\right)$ and $\Psi=\left(\Psi_{i j}\right)$ the matrices associated with the bilinear forms $\langle\cdot, \cdot\rangle_{1}$ and $\langle\cdot, \cdot\rangle_{2}$, so that we have $\left\langle q_{i j}, q_{k l}\right\rangle=\Phi_{i k} \Psi_{j l}$ for the canonical basis $q_{i j}$ of $W=W_{1} \otimes W_{2}$. In particular, these $q_{i j}$ 's satisfy the commutation relation $\left\{q_{i j}, q_{k l}\right\}=\left\langle q_{i j}, q_{k l}\right\rangle=\Phi_{i k} \Psi_{j l}$ as the elements of the Clifford algebra $\mathscr{C}(W)$. Consider the $m \times n$ matrices $Q=\left(q_{i j}\right)$ and $Q^{*}=\left(q_{i j}^{*}\right)=\Phi^{-1} Q^{\prime} \Psi^{-1}$, so that the commutation relation $\left\{q_{i j}^{*}, q_{k l}\right\}=\delta_{i k} \delta_{j l}$ holds. Then the actions of $\mathfrak{g}$ and $\mathfrak{g}^{\prime}$ are given as follows ([6]):

$$
\begin{gathered}
\sigma(F)=Q \Psi^{-1}{ }^{t} Q^{\prime} \Phi^{-1}-(n / 2) I_{m}=Q^{t} Q^{*}-(n / 2) I_{m}, \\
\sigma\left(F^{\prime}\right)={ }^{\prime} Q \Phi^{-1} Q^{t} \Psi^{-1}-(m / 2) I_{n}={ }^{\prime} Q Q^{*}-(m / 2) I_{n} .
\end{gathered}
$$

In particular, Theorem 5.1 is rewritten as

$$
\operatorname{tr}\left(Q^{\prime} Q^{*}\left(Q^{\prime} Q^{*}-(m-\varepsilon / 2) I_{m}\right)^{r}\right)=(-)^{r} \operatorname{tr}\left({ }^{\prime} Q Q^{*}\left({ }^{\prime} Q Q^{*}-(n-\varepsilon / 2) I_{n}\right)^{r}\right) .
$$


This equality can be deduced in a way similar to the proof of Theorem 3.1 by combining the following fundamental relations:

LEMMA 5.2. The following equalities hold:

$$
\left.{ }^{\prime} Q^{*} Q-m I_{n}=-{ }^{\prime}{ }^{t} Q Q^{*}\right), \quad Q^{*} Q-n I_{m}=-{ }^{\prime}\left(Q^{\prime} Q^{*}\right) .
$$

LEMMA 5.3. The entries of $Q^{\prime} Q^{*}$ and ${ }^{t} Q Q^{*}$ mutually commute:

$$
\left[\left(Q^{t} Q^{*}\right)_{i j},\left({ }^{t} Q Q^{*}\right)_{k l}\right]=0 .
$$

LEMMA 5.4. The following equality holds:

$$
\left(Q^{\prime}\left({ }^{t} Q Q^{*}-(\varepsilon / 2) I_{n}\right)\right)_{i j}=-\sum_{s=1}^{m} q_{s j}\left(Q^{t} Q^{*}-(\varepsilon / 2) I_{m}\right)_{i s} .
$$

Proposition 5.5. For any $Z \in \operatorname{Mat}_{m, n}(\mathscr{C}(W))$, the following equality holds:

$$
\begin{aligned}
& \operatorname{tr}\left(Q^{t}\left({ }^{\prime} Q Q^{*}-(\varepsilon / 2) I_{n}\right)^{r} \cdot{ }^{t} Z\right) \\
& \quad=-\operatorname{tr}\left(Q^{t}\left({ }^{t} Q Q^{*}-(\varepsilon / 2) I_{n}\right)^{r-1} \cdot{ }^{t}\left({ }^{t}\left(Q^{t} Q^{*}-(\varepsilon / 2) I_{m}\right) Z\right)\right) .
\end{aligned}
$$

COROLlaRY 5.6. For any $Z \in \operatorname{Mat}_{m, n}(\mathscr{C}(W))$, the following equality holds:

$$
\begin{aligned}
\operatorname{tr}\left(Q^{t}\left({ }^{t} Q Q^{*}-(\varepsilon / 2) I_{n}\right)^{r} \cdot{ }^{t} Z\right) & =(-)^{r} \operatorname{tr}\left(Q \cdot{ }^{t}\left({ }^{t}\left(Q^{t} Q^{*}-(\varepsilon / 2) I_{m}\right)^{r} Z\right)\right) \\
& =(-)^{r} \operatorname{tr}\left({ }^{t} Q^{t}\left(Q^{t} Q^{*}-(\varepsilon / 2) I_{m}\right)^{r} Z\right) .
\end{aligned}
$$

\section{References}

[1] A. Capelli, 'Über die Zurückführung der Cayley'schen Operation $\Omega$ auf gewöhnliche PolarOperationen', Math. Ann. 29 (1887), 331-338.

[2] —_, 'Sur les opérations dans la théorie des formes algébriques', Math. Ann. 37 (1890), 1-37.

[3] I. M. Gelfand, 'Center of the infinitesimal groups', Mat. Sbornik N. S. 26 (1950), 103-112; English translation: Collected papers Vol. II, pp. 22-30.

[4] R. Howe, ' $\theta$-series and invariant theory', in: Automorphic forms, representations, and L-functions. Part I (eds. A. Borel and W. Casselman), Proc. Sympos. Pure Math. XXXIII (Amer. Math. Soc., Providence, 1979) pp. 275-285.

[5] —_, 'Remarks on classical invariant theory', Trans. Amer. Math. Soc. 313 (1989), 539-570; Erratum in: Trans. Amer. Math. Soc. 318 (1990) 823.

[6] _- 'Perspectives on invariant theory: Schur duality, multiplicity-free actions and beyond', in: The Schur lectures (1992), Israel Math. Conf. Proc. 8 (Bar-Ilan Univ., Ramat Gan, 1995) pp. $1-182$. 
[7] R. Howe and T. Umeda, 'The Capelli identity, the double commutant theorem, and multiplicity-free actions', Math. Ann. 290 (1991), 565-619.

[8] M. Itoh, 'Explicit Newton's formulas for $\mathfrak{g l}_{n}$ ', J. Algebra 208 (1998), 687-697.

[9] M. Kashiwara and M. Vergne, 'On the Segal-Shale-Weil representation and harmonic polynomial', Invent. Math. 44 (1978), 1-47.

[10] W. H. Klink and T. Ton-That, 'On resolving the multiplicity of arbitrary tensor products of the $U(N)$ groups', J. Phys. A 21 (1988), 3877-3892.

[11] E. Y. Leung, 'On resolving the multiplicity of tensor products of irreducible representations of symplectic groups', J. Phys. A 26 (1993), 5851-5866.

[12] E. Y. Leung and T. Ton-That, 'Invariant theory of the dual pairs $\left(S O^{*}(2 n), S p(2 k, C)\right)$ and $(\operatorname{Sp}(2 n, \mathfrak{R}), O(N))^{\prime}$, Proc. Amer. Math. Soc. $120(1994), 53-65$.

[13] J. D. Louck and L. C. Biedenharn, 'Canonical unit adjoint tensor operators in $U(n)$ ', J. Math. Phys. 11 (1970), 2368-2414.

[14] C. Mœglin, M.-F. Vignéras and J.-L. Waldspurger, Correspondances de Howe sur un corps padique, Lecture Notes in Math. 1291 (Springer, Berlin, 1987).

[15] A. Molev, 'Sklyanin determinant, Laplace operators, and characteristic identities for classical Lie algebras', J. Math. Phys. 36 (1995), 923-943.

[16] A. Molev and M. Nazarov, 'Capelli identities for classical Lie algebras', Math. Ann. 313 (1999), 315-357.

[17] M. Nazarov, 'Quantum Berezinian and the classical Capelli identity', Lett. Math. Phys. 21 (1991), 123-131.

[18] A. M. Perelomov and V. S. Popov, 'Casimir operators for $U(n)$ and $S U(n)$ ', Soviet J. Nuclear Phys. 3 (1966), 676-680.

[19] _ 'Casimir operators for the orthogonal and symplectic groups', Soviet J. Nuclear Phys. 3 (1966), 819-824.

[20] M. Schmidt, 'Classification and partial ordering of reductive Howe dual pairs of classical Lie groups', J. Geom. Phys. 29 (1999), 283-318.

[21] T. Umeda, 'The Capelli identities, a century after', Sugaku 46 (1994), 206-227 (in Japanese); English translation: Selected papers on harmonic analysis, groups, and invariants (ed. K. Nomizu) Amer. Math. Soc. Transl. Ser. 2, 183 (Amer. Math. Soc., Providence, RI, 1998) pp. 51-78.

[22] _- 'Newton's formula for $\mathfrak{g l}_{n}$ ', Proc. Amer. Math. Soc. 126 (1998), 3169-3175.

[23] D. P. Želobenko, Compact Lie groups and their representations, Transl. Math. Monographs 40 (Amer. Math. Soc., Providence, 1973).

Department of Mathematics

Faculty of Science

Kyoto University

Kyoto 606-8502

Japan

e-mail: minoru@kusm.kyoto-u.ac.jp 
J. Aust. Math. Soc. 75 (2003) 